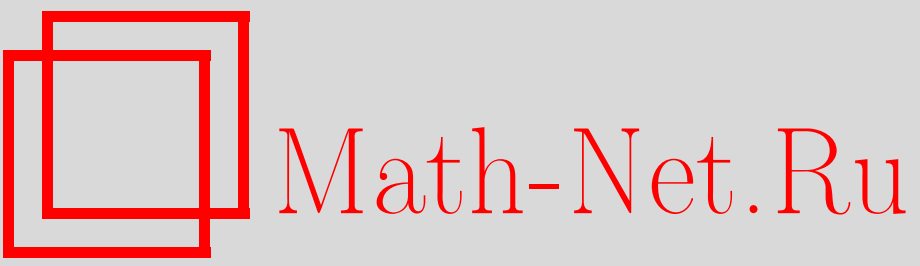

А. А. Жукова, А. В. Шутов, Аддитивная задача с $k$ числами специального вида, Итоги науки и техн. Сер. Соврем. мат. и ее прил. Темат. обз., 2019, том 166, 10-21

DOI: https://doi.org/10.36535/0233-6723-2019-166-10-21

Использование Общероссийского математического портала Math-Net.Ru подразумевает, что вы прочитали и согласны с пользовательским соглашением

http://www . mathnet.ru/rus/agreement

Параметры загрузки:

IP: 54.166 .219 .16

26 апреля 2023 г., 17:20:23 


\title{
АДДИТИВНАЯ ЗАДАЧА С $k$ ЧИСЛАМИ СПЕЦИАЛЬНОГО ВИДА
}

\author{
(c) 2019 г. A. А. ЖУКОВА, А. В. ШУТОВ
}

\begin{abstract}
АннотАция. В работе рассматривается аддитивная задача вида $n_{1}+n_{2}+\ldots+n_{k}=N$ с числом слагаемых не менее двух и с условиями на слагаемые $n_{i} \in \mathbb{N}\left(\alpha_{i}, I_{i}\right)$ при $1 \leqslant i \leqslant k$, где $\mathbb{N}(\alpha, I)=$ $\{n \in \mathbb{N}:\{n \alpha\} \in I\}$.
\end{abstract}

Ключевъе слова: аддитивная задача, равномерное распределение.

\section{ADDITIVE PROBLEM WITH $k$ NUMBERS OF A SPECIAL FORM}

\section{(c) 2019 A. A. ZHUKOVA, A. V. SHUTOV}

\begin{abstract}
In this paper, we consider an additive problem of the form $n_{1}+n_{2}+\ldots+n_{k}=N$ with at least two summands, where the summands satisfy the condition $n_{i} \in \mathbb{N}\left(\alpha_{i}, I_{i}\right)$ for $1 \leqslant i \leqslant k$, where $\mathbb{N}(\alpha I)=\{n \in \mathbb{N}:\{n \alpha\} \in I\}$.
\end{abstract}

Keywords and phrases: additive problem, uniform distribution.

AMS Subject Classification: 11P99

1. Введение. В работе рассматривается аддитивная задача вида $n_{1}+n_{2}+\ldots+n_{k}=N$ с числом слагаемых не менее двух и с условиями на слагаемые $n_{i} \in \mathbb{N}\left(\alpha_{i}, I_{i}\right)$ при $1 \leqslant i \leqslant k$, где $\mathbb{N}(\alpha, I)=$ $\{n \in \mathbb{N}:\{n \alpha\} \in I\}$. Такие множества описывают, в частности, натуральные числа, имеющие заданное окончание разложения по линейным рекуррентным последовательностям, связанным с квадратичными числами Пизо. Кроме того, множества $\mathbb{N}(\alpha, I)$ являются частными случаями так называемых квазирешеток. Ранее рассматривались аддитивные задачи на множествах такого вида для случая $\alpha_{i}=\alpha$ при всех $i$. В этом случае были получены асимптотические формулы для числа решений аддитивной задачи с произвольным числом слагаемых и для среднего числа решений этой задачи. С. А. Гриценко и Н. Н. Мотькина обнаружили, что в случае линейных задач возникает нетривиальный эффект: появление некоторой достаточно сложной функции в главном члене асимптотики числа решений. Для нелинейных задач подобный эффект отсутствует и вид главного члена получается из соображений плотности.

В рассматриваемой задаче обнаружено, что поведение главного члена асимптотической формулы для числа решений существенным образом зависит от арифметических свойств $\alpha_{i}$. В случае, когда размерность векторного пространства над полем рациональных чисел, порожденного числами $1, \alpha_{1}, \ldots, \alpha_{k}$, равна двум, имеет место эффект Гриценко-Мотькиной, т.е. главный член имеет вид $\rho_{k}\left(\left\{N \hat{\alpha}_{k}\right\}\right) N^{k-1}$, где $\rho_{k}(x)$ - эффективно вычислимый кусочный многочлен степени $k-1$ и $\hat{\alpha}_{k}$ эффективно выражается через $\alpha_{1}, \ldots, \alpha_{k}$. В остальных случаях главный член асимптотики имеет плотностный вид, т.е. равен $\frac{1}{(k-1) !} \prod_{i=1}^{k}\left|I_{i}\right| \cdot N^{k-1}$.

Пусть $\alpha$-иррациональное число и $I \subset[0 ; 1)$ - некоторый интервал. Определим множество

$$
\mathbb{N}(\alpha, I)=\{n \in \mathbb{N}:\{n \alpha\} \in I\},
$$


где $\{\cdot\}$ означает дробную долю числа.

Впервые частный случай чисел вида $\mathbb{N}(\alpha, I)$ возник в [11]. Пусть $F_{n}-n$-е число Фибоначчи, т.е. элемент последовательности, заданной рекуррентной формулой $F_{n}=F_{n-1}+F_{n-2}, F_{1}=1$, $F_{2}=2$. Любое натуральное число $N$ может быть однозначно разложено в систему счисления Фибоначчи, т.е.

$$
N=\sum_{k \geqslant 1} \varepsilon_{k}(N) F_{k}, \quad \text { причем } \varepsilon_{k}(N) \varepsilon_{k+1}(N)=0 .
$$

Пусть $\mathbb{F}_{0}=\left\{N \in \mathbb{N}: \varepsilon_{1}(N)=0\right\}$ - множество четно-фибоначчевых чисел. В [11] фактически было показано, что $\mathbb{F}_{0}=\mathbb{N}\left(\tau, I_{1}\right) \cup \mathbb{N}\left(\tau, I_{2}\right)$, где $\tau=(\sqrt{5}-1) / 2$, вычислены интервалы $I_{1}$ и $I_{2}$. Данное описание множества $\mathbb{F}_{0}$ позволило решить над этим множеством целый ряд теоретикочисловых задач. Были получены асимптотическая формула для решения бинарной аддитивной задачи в числах из $\mathbb{F}_{0}$, асимптотическая формула для количества чисел из $\mathbb{F}_{0}$, принадлежащих заданной арифметической прогрессии, оценки тригонометрических сумм по числам из $\mathbb{F}_{0}$.

В дальнейшем описанная характеризация была обобщена на случай чисел, имеющих произвольное заданное окончание разложения в систему счисления Фибоначчи (см. [7]). Аналогичный результат был получен для систем счисления, порождаемых рекуррентными соотношениями вида

$$
F_{i+2}^{(g)}=g F_{i+1}^{(g)}+F_{i}^{(g)}
$$

(см. [8]). Кроме того, подобные результаты были получены для некоторых специальных систем счисления, возникающих при изучении множеств ограниченного остатка (см. [20]).

В [1-6] было найдено решение целого ряда теоретико-числовых задач над числами $\mathbb{N}(\alpha, I)$ в случае, когда $\alpha$ является квадратичной иррациональностью либо алгебраическим числом. В частности, были найдены решения аналогов тернарной проблемы Гольдбаха (см. [4]), проблемы ХуаЛокена (см. [1]), проблемы Варинга (см. [6]), проблемы Лагранжа о четырех квадратах (см. [3]), а также получена оценка мощности исключительного множества для аналога бинарной проблемы Гольдбаха (см. [5]). При этом был обнаружен следующий эффект. В случае нелинейных аддитивных задач удавалось доказать асимптотические формулы вида $r(N) \sim|I|^{k} r^{*}(N)$, где $r(N)-$ число решений нелинейной аддитивной задачи с дополнительным условием принадлежности слагаемых множествам $\mathbb{N}(\alpha, I), r^{*}(N)$ - число решений нелинейной аддитивной задачи без данного условия, а $k$ - число слагаемых. В случае линейных задач асимптотическая формула принимает вид $r(N) \sim \sigma(N) r^{*}(N)$, где $\sigma(N)$ - некоторый особый ряд.

Бинарная аддитивная задача вида

$$
n_{1}+n_{2}=N
$$

с дополнительными условиями

$$
n_{1} \in \mathbb{N}\left(\alpha_{1}, I_{1}\right), \quad n_{2} \in \mathbb{N}\left(\alpha_{2}, I_{2}\right)
$$

и произвольными иррациональными $\alpha_{1}, \alpha_{2}$ решена в [9].

В данной работе найдена асимптотическая формула для числа решений уравнения

$$
n_{1}+\ldots+n_{k}=N \text {, }
$$

где $k \geqslant 2$, слагаемые удовлетворяют условиям

$$
n_{i} \in \mathbb{N}\left(\alpha_{i}, I_{i}\right) \quad \text { при всех } 1 \leqslant i \leqslant k,
$$

$\alpha_{i}$ - произвольные иррациональные числа.

В [19] была рассмотрена аддитивная задача о числе решений уравнения (3) с дополнительными условиями на слагаемые $n_{i} \in \mathbb{N}\left(\alpha, I_{i}\right)$, с произвольным количеством слагаемых $k$ и произвольным иррациональным $\alpha$. Была получена асимптотическая формула для числа решений рассматриваемой задачи, в которой особый ряд был заменен эффективно вычислимым интегралом кратности $k-1$. В частности, была решена проблема необращения особого ряда в нуль. Альтернативная конечная формула для особого ряда была найдена в [2].

Отметим, что рассматриваемые нами множества $\mathbb{N}(\alpha, I)$ являются примерами так называемых одномерных квазирешеток. Более общий взгляд на одномерные квазирешетки можно найти 
в $[12,18]$. Многочисленные примеры рассмотрения теоретико-числовых задач над квазирешетками различных типов могут быть найдены в $[10,13,15-17,21]$.

2. Вспомогательные результаты. Для произвольного $\varepsilon$ определим отображение

$$
\iota_{\varepsilon}: x \rightarrow 1-x+\varepsilon \quad(\bmod 1) .
$$

Лемма 1. Если $N-n_{1}=n$, где $n, n_{1}, N$-натуральные числа и $n_{1}<N$, то условие $\left\{\left(N-n_{1}\right) \alpha\right\} \in I$ эквивалентно условию $\left\{n_{1} \alpha\right\} \in I^{\prime}(N)$, где $I^{\prime}(N)=\iota_{\{N \alpha\}}(I)$.

Доказательство. Пусть $I=(a, b)$. С одной стороны, условие $\left\{\left(N-n_{1}\right) \alpha\right\} \in I$ эквивалентно неравенствам

при $\{N \alpha\} \geqslant\left\{n_{1} \alpha\right\}$ и

$$
\{N \alpha\}-b<\left\{n_{1} \beta\right\}<\{N \alpha\}-a
$$

при $\{N \alpha\}<\left\{n_{1} \alpha\right\}$.

$$
\{N \alpha\}+1-b<\left\{n_{1} \alpha\right\}<\{N \alpha\}+1-a
$$

С другой стороны, согласно определению отображения

$$
\iota_{\{N \alpha\}}: x \rightarrow 1-x+\{N \alpha\} \quad(\bmod 1)
$$

имеем

$$
a \rightarrow 1-a+\{N \alpha\} \quad(\bmod 1), \quad b \rightarrow 1-b+\{N \alpha\} \quad(\bmod 1) .
$$

Это означает, что условия

$$
\left\{\left(N-n_{1}\right) \alpha\right\} \in I, \quad\left\{n_{1} \alpha\right\} \in \iota_{\{N \alpha\}}(I)
$$

равносильны. Полагая $I^{\prime}(N)=\iota_{\{N \alpha\}}(I)$, получим утверждение леммы 1 .

Лемма 2 (см. [25]). Пусть $f$-интегрируемая по Риману функиия, $\alpha$ - иррациональное число. Тогда

$$
\sum_{i=1}^{n} f(\{i \alpha\}) \sim n \int_{0}^{1} f(t) d t .
$$

Пусть $\chi_{I}(x)$ - характеристическая функция интервала $I \subseteq[0 ; 1)$, т.е.

$$
\chi_{I}(x)= \begin{cases}1, & \text { если } x \in I, \\ 0, & \text { если } x \notin[0 ; 1) \backslash I ;\end{cases}
$$

$\chi_{I}(x)$ продолжается до периодической функции с периодом 1.

Лемма 3. При любом натуральном $n, I \subseteq[0,1)$, справедлива асимптотика

$$
\sum_{i=1}^{n} \chi_{I}(\{i \alpha\}) \sim n|I|
$$

где $|I|-$ длина интервала $I$.

Доказательство. Характеристическая функция $\chi_{I}(x)$ является кусочно постоянной, поэтому удовлетворяет условиям леммы 2. Имеем

$$
\sum_{i=1}^{n} \chi_{I}(\{i \alpha\}) \sim n \int_{0}^{1} \chi_{I}(t) d t=n \cdot|I| .
$$

Лемма 4. При любом натуральном $n$, иррачиональном $\alpha u I \subseteq[0,1)$ справедлива асимптотическая формула

где $|I|$ - длина интервала $I$.

$$
\sum_{n=1}^{M} n^{m-1} \chi_{I}(\{n \alpha\}) \sim \frac{|I|}{m} M^{m},
$$


Доказательство. Воспользуемся формулой суммирования Абеля

$$
\sum_{n=1}^{M} a_{n} b_{n}=a_{M} B_{M}-\sum_{n=1}^{M-1}\left(a_{n+1}-a_{n}\right) B_{n}, \quad \text { где } \quad B_{n}=\sum_{i=1}^{n} b_{i},
$$

положив

$$
a_{n}=n^{m-1}, \quad b_{n}=\chi_{I}(\{n \alpha\}), \quad B_{n}=\sum_{i=1}^{n} \chi_{I}(\{i \alpha\}) .
$$

Согласно лемме 3 сумма $B_{n}$ асимптотически равна $n|I|$. В таком случае

$$
\begin{aligned}
\sum_{n=1}^{M} n^{m-1} \chi_{I}(\{n \alpha\}) & \sim M^{m-1} B_{M}-\sum_{n=1}^{M-1}\left((n+1)^{m-1}-n^{m-1}\right) B_{n}= \\
& =M^{m-1} \cdot M|I|-\sum_{n=1}^{M-1}(n+1-n) \sum_{j=0}^{m-2}(n+1)^{j} n^{m-2-j} \cdot n|I|= \\
& =|I|\left(M^{m}-\sum_{n=1}^{M-1} n^{m-1} \sum_{j=0}^{m-2}\left(1+\frac{1}{n}\right)^{j}\right) \sim|I|\left(M^{m}-(m-1) \sum_{n=1}^{M-1} n^{m-1}\right) .
\end{aligned}
$$

Далее, с учетом асимптотической формулы

$$
\sum_{n=1}^{M} n^{m} \sim \frac{1}{m+1} M^{m+1}
$$

имеем

$$
\sum_{n=1}^{M} n^{m-1} \chi_{I}(\{n \alpha\}) \sim|I|\left(M^{m}-(m-1) \frac{(M-1)^{m}}{m}\right) \sim|I|\left(M^{m}-\frac{m-1}{m} M^{m}\right)=\frac{|I|}{m} M^{m} .
$$

Пусть $0<\alpha<1,0<\beta<1$ и $1, \alpha, \beta$-линейно зависимые над $\mathbb{Z}$ величины, т.е. существуют такие целые числа $C_{1}, C_{2}, C_{3}$, одновременно не равные нулю, что $C_{1} \alpha+C_{2} \beta+C_{3}=0$. Обозначим через $\operatorname{Orb}(\alpha, \beta)$ замыкание множества точек вида $\left\{Q_{i}=(\{i \alpha\},\{i \beta\})\right\}$, где $i \in \mathbb{N}$, и назовем орбитой точки $Q_{i}$.

Лемма 5 (см. [9]). Длина замыкания орбиты $\operatorname{Orb}(\alpha, \beta)$ вычисляется по формуле

$$
|\operatorname{Orb}(\alpha, \beta)|= \begin{cases}\frac{\left|C_{2}\right| \sqrt{1+k^{2}}}{\text { НОД }\left(\left|C_{1}\right|,\left|C_{2}\right|\right)} & \text { при } k<1, \\ \sqrt{2} & \text { при } k=1, \\ \frac{\left|C_{1}\right| \sqrt{1+k^{2}}}{k \cdot \text { НОД }\left(\left|C_{1}\right|,\left|C_{2}\right|\right)} & \text { при } k>1,\end{cases}
$$

где величины $1, \alpha, \beta$ линейно зависимы над $\mathbb{Z}$, т.е. существуют такие челье числа $C_{1}, C_{2}, C_{3}$, одновременно не равные нулю, что $C_{1} \alpha+C_{2} \beta+C_{3}=0,0<\alpha<1,0<\beta<1, k=-C_{1} / C_{2}$.

Пусть

$$
\hat{\alpha}_{m}=\frac{\left|\left(\hat{\alpha}_{m-1}, \alpha_{m}\right)\right|}{\left|\operatorname{Orb}\left(\hat{\alpha}_{m-1}, \alpha_{m}\right)\right|},
$$

где $\left(\hat{\alpha}_{m-1}, \alpha_{m}\right)$ - вектор и $\hat{\alpha}_{1}=\alpha_{1}$. Справедлива следующая лемма.

Лемма 6. Если все $\alpha_{i}$ иррачиональны, $0<\alpha_{i}<1$, и любая тройка чисел вида $1, \alpha_{i}, \alpha_{j}$ является линейно зависимой над $\mathbb{Z}$, то при любом $m \geqslant 1$ число $\hat{\alpha}_{m}$ иррачионально, а величинь 1 , $\hat{\alpha}_{m}, \alpha_{m+1}$ линейно зависимь над $\mathbb{Z}$. 
Доказательство. Проведем доказательство, используя метод математической индукции. При $m=1$ величины $1, \hat{\alpha}_{1}, \alpha_{2}$ (или иначе $1, \alpha_{1}, \alpha_{2}$ ) линейно зависимы над $\mathbb{Z}$, а $\alpha_{1}$ - иррационально по условию.

Предположим, что утверждение леммы верно при $m=l$, т.е. величины $1, \hat{\alpha}_{l}, \alpha_{l+1}$ линейно зависимы над $\mathbb{Z}$ и число $\hat{\alpha}_{l}$ иррационально. В предположении, что лемма верна при $m=l$, докажем ее справедливость при $m=l+1$. Вначале убедимся, что число

$$
\hat{\alpha}_{l+1}=\frac{\left|\left(\hat{\alpha}_{l}, \alpha_{l+1}\right)\right|}{\left|\operatorname{Orb}\left(\hat{\alpha}_{l}, \alpha_{l+1}\right)\right|}
$$

иррационально. Утверждение леммы 5 можно переписать следующим образом: длина замыкания орбиты $\operatorname{Orb}(\alpha, \beta)$ при любом $k>0$ вычисляется по формуле

$$
|\operatorname{Orb}(\alpha, \beta)|=\frac{\sqrt{C_{1}^{2}+C_{2}^{2}}}{\text { НОД }\left(\left|C_{1}\right|,\left|C_{2}\right|\right)} .
$$

Поэтому, если существуют такие целые числа $C_{1}, C_{2}, C_{3}$, одновременно не равные нулю, что $C_{1} \hat{\alpha}_{l}+C_{2} \alpha_{l+1}+C_{3}=0$, то

$$
\left|\operatorname{Orb}\left(\hat{\alpha}_{l}, \alpha_{l+1}\right)\right|=\frac{\sqrt{C_{1}^{2}+C_{2}^{2}}}{\operatorname{HОД}\left(\left|C_{1}\right|,\left|C_{2}\right|\right)} .
$$

Согласно предположению число $\hat{\alpha}_{l}$ иррационально, а $\alpha_{l+1}$ иррационально по условию; следовательно, существуют такие целые числа $C_{1}, C_{2}$, одновременно не равные нулю, что

$$
C_{1} \hat{\alpha}_{l}+C_{2} \alpha_{l+1}=0
$$

т.e.

$$
\hat{\alpha}_{l}=-\frac{C_{2}}{C_{1}} \alpha_{l+1} .
$$

В таком случае длина вектора $\left(\hat{\alpha}_{l}, \alpha_{l+1}\right)$ равна

$$
\begin{gathered}
\left|\left(\hat{\alpha}_{l}, \alpha_{l+1}\right)\right|=\sqrt{\hat{\alpha}_{l}^{2}+\alpha_{l+1}^{2}}=\sqrt{\left(-\frac{C_{2}}{C_{1}} \alpha_{l+1}\right)^{2}+\alpha_{l+1}^{2}}=\frac{\sqrt{C_{1}^{2}+C_{2}^{2}}}{\left|C_{1}\right|} \alpha_{l+1}, \\
\hat{\alpha}_{l+1}=\frac{\sqrt{C_{1}^{2}+C_{2}^{2}}}{\left|C_{1}\right|} \alpha_{l+1} / \frac{\sqrt{C_{1}^{2}+C_{2}^{2}}}{\text { НОД }\left(\left|C_{1}\right|,\left|C_{2}\right|\right)}=\frac{\text { НОД }\left(\left|C_{1}\right|,\left|C_{2}\right|\right)}{\left|C_{1}\right|} \alpha_{l+1},
\end{gathered}
$$

т.е. число $\hat{\alpha}_{l+1}=\frac{\text { НОД }\left(\left|C_{1}\right|,\left|C_{2}\right|\right)}{\left|C_{1}\right|} \alpha_{l+1}$ иррационально.

Линейная зависимость над $\mathbb{Z}$ величин $1, \hat{\alpha}_{l+1}, \alpha_{l+2}$ (или иначе чисел $1, \frac{\operatorname{HОД}\left(\left|C_{1}\right|,\left|C_{2}\right|\right)}{\left|C_{1}\right|} \alpha_{l+1}$, $\left.\alpha_{l+2}\right)$ следует из того, что по условию любая тройка чисел вида $1, \alpha_{i}, \alpha_{j}$ является линейно зависимой над $\mathbb{Z}$.

Пусть $r_{2}\left(\alpha_{1}, \alpha_{2}, I_{1}, I_{2}, N\right)$ (или, короче, $\left.r_{2}(N)\right)$ - число решений уравнения (1) с дополнительными условиями (2). В [9] доказано следующее утверждение.

Лемма 7. Справедливы асимптотические формуль

$$
r\left(\alpha_{1}, \alpha_{2}, I_{1}, I_{2}, N\right) \sim \frac{\left|\operatorname{Orb}\left(\alpha_{1}, \alpha_{2}\right) \cap P_{N}\right|}{\left|\operatorname{Orb}\left(\alpha_{1}, \alpha_{2}\right)\right|} N, \quad r\left(\alpha_{1}, \alpha_{2}, I_{1}, I_{2}, N\right) \sim \frac{\left|\operatorname{Orb}\left(\alpha_{1}, \alpha_{2}\right) \cap P_{N}^{\prime}\right|}{\left|\operatorname{Orb}\left(\alpha_{1}, \alpha_{2}\right)\right|} N
$$

где $|\cdot|$-естественная мера на множестве $\operatorname{Orb}\left(\alpha_{1}, \alpha_{2}\right), P_{N}=I_{1} \oplus \iota_{\left\{N \alpha_{2}\right\}}\left(I_{2}\right), P_{N}^{\prime}=\iota_{\left\{N \alpha_{1}\right\}}\left(I_{1}\right) \oplus I_{2}$ $u \iota_{c}(x)=\{1-x+c\}$.

Обозначим дробь $\frac{\left|\operatorname{Orb}\left(\alpha_{1}, \alpha_{2}\right) \cap P_{N}\right|}{\left|\operatorname{Orb}\left(\alpha_{1}, \alpha_{2}\right)\right|}$, стоящую в правой части первой асимптотической формулы леммы 7 , через $\rho_{2}\left(\left\{N \alpha_{2}\right\}\right)$ и докажем несколько свойств этой функции в случае линейной зависимости $\alpha_{1}$ и $\alpha_{2}$.

Лемма 8. Если $\alpha_{1}$ и $\alpha_{2}$ линейно зависимы над $\mathbb{Z}$, то функиия $\rho_{2}\left(\left\{N \alpha_{2}\right\}\right)$ является непрерывной. 
Доказательство. Согласно определению $\rho_{2}\left(\left\{N \alpha_{2}\right\}\right)$ - это отношение длины орбиты точки $\left(\alpha_{1}, \alpha_{2}\right)$, попадающей в прямоугольник $P_{N}$, к длине всей орбиты. В силу леммы 5 величина $\left|\operatorname{Orb}\left(\alpha_{1}, \alpha_{2}\right)\right|$ является фиксированной для данных $\alpha_{1}$ и $\alpha_{2}$.

Из линейной зависимости чисел $\alpha_{1}$ и $\alpha_{2}$ следует, что орбита точки $\left(\alpha_{1}, \alpha_{2}\right)$ представляет собой отрезки параллельных прямых, расположенных на равных расстояниях, заключенных в единичном квадрате.

При изменении $N$ меняется положение прямоугольника $P_{N}=I_{1} \oplus \iota_{\left\{N \alpha_{2}\right\}}\left(I_{2}\right)$, но не его размеры, причем происходит сдвиг прямоугольника вдоль одной из осей координат (для определенности будем считать, что вдоль горизонтальной оси $O x$ ). При сдвиге прямоугольника $P_{N}$ вдоль оси $O x$ на бесконечно малую величину, разность между длиной орбиты точки $\left(\alpha_{1}, \alpha_{2}\right)$, попадающей в «новый» и в «старый» прямоугольники, будет бесконечно мала в силу равномерности расположения отрезков прямых $\operatorname{Orb}\left(\alpha_{1}, \alpha_{2}\right)$. Это означает, что функция $\rho_{2}\left(\left\{N \alpha_{2}\right\}\right)$ является непрерывной.

Лемма 9. В случае линейной зависимости $\alpha_{1}$ и $\alpha_{2}$ над $\mathbb{Z}$ функиия $\rho_{2}(x)$ представляет собой кусочно линейную периодическую функцию.

Данное утверждение является следствием из лемм 5 и 6 , доказанных в работе [9].

Воспользуемся перечисленными свойствами для доказательства еще одного свойства функции $\rho_{2}(x)$.

Лемма 10. Если $\alpha_{1}$ и $\alpha_{2}$ линейно зависимь над $\mathbb{Z}$, то график функиии $\rho_{2}(x)$ состочт из отрезков прямых, из которых хотя бы две имеют различные угловые коэффичиентыь.

Доказательство. Предположим противное: пусть непрерывная и $c$-периодическая кусочно линейная функция $\rho_{2}(x)$ состоит из прямых с одинаковым угловым коэффициентом. Возможны два случая:

(1) функция $\rho_{2}(x)$ представляет собой отрезок только одной прямой, т.е. $\rho_{2}(x)=a_{1} x+a_{0}$;

(2) функция $\rho_{2}(x)$ состоит по крайней мере из двух прямых с одинаковым угловым коэффициентом.

Функция $\rho_{2}(x)$ периодическая, то есть существует $c>0$, такое что $\rho_{2}(x+c)=\rho_{2}(x)$ для любых $x$. Очевидно, что в первом случае $\rho_{2}(0) \neq \rho_{2}(c)$, следовательно, нарушается свойство периодичности функции $\rho_{2}(x)$.

Во втором случае найдутся по крайней мере два таких отрезка $\left[t_{i-1} ; t_{i}\right]$ и $\left[t_{i} ; t_{i+1}\right] \subset[0 ; c]$, что $\rho_{2}(x)=a_{1} x+a_{i}$, если $x \in\left[t_{i-1} ; t_{i}\right] ; \rho_{2}(x)=a_{1} x+a_{i+1}$, если $x \in\left[t_{i} ; t_{i+1}\right]$, причем $a_{i} \neq a_{i+1}$.

В таком случае при $x=t_{i}$ нарушается свойство непрерывности функции $\rho_{2}(x)$, так как $a_{1} t_{i}+$ $a_{i} \neq a_{1} t_{i}+a_{i+1}$.

Значит, предположение о том, что непрерывная периодическая кусочно линейная функция $\rho_{2}(x)$ состоит из прямых с одинаковым угловым коэффициентом, неверно.

Основным результатом работы [9] является следующее утверждение.

Лемма 11. Справедлива асимптотическая формула

$$
r_{2}\left(\alpha_{1}, \alpha_{2}, I_{1}, I_{2}, N\right) \sim \begin{cases}\left|I_{1}\right|\left|I_{2}\right| N, & \text { если } 1, \alpha_{1}, \alpha_{2} \text { линейно независимы над } \mathbb{Z}, \\ \rho_{2}\left(\left\{N \alpha_{2}\right\}\right) N, & \text { если } 1, \alpha_{1}, \alpha_{2} \text { линейно зависимы над } \mathbb{Z} ;\end{cases}
$$

здесъ $\rho_{2}(x)$ представляет собой эффективно вычислимую кусочно линейную функцию, явный вид которой зависит от расположения интервалов $I_{1}, I_{2}$, а такэе от вида линейной зависимости между $1, \alpha_{1} u \alpha_{2}$.

Введем обозначение

$$
\rho_{m+1}(x)=\int_{0}^{1} \hat{\rho}_{m}(t) \chi_{I}(x-t) d t
$$

где $m \geqslant 2$, и докажем следующую лемму. 
Лемма 12. Если $\hat{\rho}_{m}(x)$ - непрерывная периодическая функиия с периодом 1, представляющая собой кусочный многочлен степени $m-1$, причем по крайней мере два многочлена имеют различные стариие коэфбиииенты, то $\rho_{m+1}(x)$ такэне будет непрерьвной периодической функиией с периодом 1, являющейся таким кусочным многочленом степени т, что хотя бы два многочлена имеют различные старшие коэфбиииенты.

Доказательство. Опираясь на лемму 1, можно утверждать, что значения функции $\chi_{I}(x-t)$ совпадают со значениями функции $\chi_{I^{\prime}(x)}(t)$, где $I^{\prime}(x)=\iota_{x}(I)$. В таком случае

$$
\rho_{m+1}(x)=\int_{0}^{1} \hat{\rho}_{l}(t) \chi_{I^{\prime}(x)}(t) d t=\int_{x}^{x+\left|I^{\prime}(x)\right|} \hat{\rho}_{m}(t) d t=\int_{x}^{x+|I|} \hat{\rho}_{m}(t) d t-\int_{0}^{x} \hat{\rho}_{m}(t) d t,
$$

так как $\left|I^{\prime}(x)\right|=|I|$.

Непрерывность функции $\rho_{m+1}(x)$ следует из непрерывности функции $\hat{\rho}_{m}(x)$ и свойств интеграла с переменным верхним пределом.

Возможны два случая: (1) $x+|I| \leqslant 1 ;(2) x+|I|>1$. В силу периодичности функции $\hat{\rho}_{m}(x)$ во втором случае имеем

$$
\rho_{m+1}(x)=\int_{0}^{1} \hat{\rho}_{m}(t) d t+\int_{0}^{x+|I|-1} \hat{\rho}_{m}(t) d t-\int_{0}^{x} \hat{\rho}_{m}(t) d t=\int_{0}^{x+|I|-1} \hat{\rho}_{m}(t) d t-\int_{0}^{x} \hat{\rho}_{m}(t) d t+C_{0} .
$$

В силу периодичности функции $\hat{\rho}_{m}(x)$ можно ограничиться определением этой функции на отрезке $[0 ; 1]$.

Отрезок $[0 ; 1]$ разобьем на части такими точками $t_{i}$, что $t_{0}=0<t_{1}<\ldots<t_{n}=1$. Если $x \in\left[t_{i-1} ; t_{i}\right]$, где $i=1,2, \ldots, n$, то

$$
\hat{\rho}_{m}(x)=P_{i, m-1}(x)=\sum_{r=0}^{m-1} a_{i, r} x^{r},
$$

причём $P_{i, m-1}(x) \neq P_{i+1, m-1}(x)$ при любых $i=1,2, \ldots, n-1$.

Рассмотрим отдельно интеграл $\int_{0}^{x+\Delta} \hat{\rho}_{m}(t) d t$, где $x+\Delta \in\left[t_{p-1} ; t_{p}\right]$. Очевидно, что

$$
\begin{gathered}
\int_{0}^{x+\Delta} \hat{\rho}_{m}(t) d t=\int_{0}^{t_{p-1}} \hat{\rho}_{m}(t) d t+\int_{t_{p-1}}^{x+\Delta} \hat{\rho}_{m}(t) d t= \\
=C_{p, 0}+\int_{t_{p-1}}^{x+\Delta} \sum_{r=0}^{m-1} a_{p, r} t^{r} d t=\left.\sum_{r=0}^{m-1} \frac{a_{p, r}}{r+1} t^{r+1}\right|_{t_{p-1}} ^{x+\Delta}+C_{p, 0}= \\
=\sum_{r=0}^{m-1} \frac{a_{p, r}}{r+1}(x+\Delta)^{r+1}-\sum_{r=0}^{m-1} \frac{a_{p, r}}{r+1} t_{p-1}^{r+1}+C_{p, 0}=\sum_{r=0}^{m-1} a_{p, r}^{\prime} x^{r+1}+C_{p}
\end{gathered}
$$

где

$$
\begin{gathered}
C_{p, 0}=\int_{0}^{t_{p-1}} \hat{\rho}_{m}(t) d t, \quad a_{p, r}^{\prime}=\sum_{s=0}^{m-r-1} \frac{(r+s+1) !}{s !(r+1) !} \frac{a_{p, r+s}}{r+s+1} \Delta^{s}, \\
C_{p}=C_{p, 0}-\sum_{r=0}^{m-1} \frac{a_{p, r}}{r+1} t_{p-1}^{r+1}+\sum_{r=0}^{m-1} \frac{a_{p, r}}{r+1} \Delta^{r+1} .
\end{gathered}
$$

Пусть $x \in\left[t_{i-1} ; t_{i}\right], x+|I|-1 \in\left[t_{j-1} ; t_{j}\right]$ и $x+|I| \in\left[t_{k-1} ; t_{k}\right]$; тогда 
(1) при $x+|I| \leqslant 1$ получаем

$$
\rho_{m+1}(x)=\sum_{r=0}^{m-1} a_{k, r}^{\prime} x^{r+1}+C_{k}-\left(\sum_{r=0}^{m-1} a_{i, r}^{\prime} x^{r+1}+C_{i}\right) ;
$$

(2) при $x+|I|>1$ имеем

$$
\rho_{m+1}(x)=C_{0}+\sum_{r=0}^{m-1} a_{j, r}^{\prime} x^{r+1}+C_{j}-\left(\sum_{r=0}^{m-1} a_{i, r}^{\prime} x^{r+1}+C_{i}\right) .
$$

По условию у функции $\hat{\rho}_{m}(x)$ найдутся хотя бы два не равных старших коэффициента многочлена $P_{i, m-1}(x)$ и $P_{j, m-1}(x)$, т.е. существуют $a_{i, m-1} \neq a_{j, m-1}$, следовательно, $a_{i, m-1}^{\prime} \neq a_{j, m-1}^{\prime}$.

Таким образом, доказано, что $\rho_{m+1}(x)$ является кусочным многочленом степени $m$, имеющим по крайней мере два различных старших коэффициента.

3. Общий случай. Пусть $r_{k}\left(\alpha_{1}, \ldots, \alpha_{k}, I_{1}, \ldots, I_{k}, N\right)=r_{k}(N)$ - число решений уравнения (3) с условиями на слагаемые (4) и $k \geqslant 2$.

Рассмотрим линейное пространство $\mathbb{A}^{n}$ над полем рациональных чисел, порожденное числами $1, \alpha_{1}, \alpha_{2}, \ldots, \alpha_{n}$, где $\alpha_{i}$ - такие иррациональные числа, что $0<\alpha_{i}<1, i=1,2, \ldots, n$. Пусть $d$ - размерность пространства $\mathbb{A}^{n}$ над $\mathbb{Q}$. В силу того, что все $\alpha_{i}$ иррациональны, выполняется неравенство $2 \leqslant d \leqslant n+1$.

Теорема 1. Справедлива асимптотическал формула

$$
r_{k}(N) \sim \begin{cases}\frac{1}{(k-1) !} \rho_{k}\left(\left\{N \hat{\alpha}_{k}\right\}\right) N^{k-1}, & \text { если } d=2, \\ \frac{1}{(k-1) !} \prod_{i=1}^{k}\left|I_{i}\right| \cdot N^{k-1}, & \text { если } d \geqslant 3\end{cases}
$$

здесь $\hat{\alpha}_{k}-$ эффективно выражсаемая через $1, \alpha_{1}, \ldots, \alpha_{k}$ величина, $\rho_{k}(x)-$ кусочный многочлен степени $k-1$ и $k \geqslant 2$.

Доказательство. Доказательство проведем методом математической индукции. Вначале убедимся в том, что теорема 1 верна при $k=2$. Действительно, при $k=2$ получаем результат, совпадающий с леммой 11. При этом можно положить $\hat{\alpha}_{2}=\alpha_{2}$.

Предположим, что теорема 1 справедлива при $k=m$, т.е. асимптотическая формула для числа решений уравнения $n_{1}+n_{2}+\ldots+n_{m}=N$ с дополнительными условиями на слагаемые $n_{i} \in$ $\mathbb{N}\left(\alpha_{i}, I_{i}\right)$ имеет вид

$$
r_{m}(N) \sim \begin{cases}\frac{1}{(m-1) !} \rho_{m}\left(\left\{N \hat{\alpha}_{m}\right\}\right) N^{m-1}, & \text { если } d=2, \\ \frac{1}{(m-1) !} \prod_{i=1}^{m}\left|I_{i}\right| \cdot N^{m-1}, & \text { если } d \geqslant 3\end{cases}
$$

здесь $\hat{\alpha}_{m}$ - эффективно выражаемая через $1, \alpha_{1}, \ldots, \alpha_{m}$ величина, $\rho_{m}(x)-$ кусочный многочлен степени $m-1$.

В предположении, что теорема верна при $k=m$, докажем её справедливость при $k=m+1$. Перепишем уравнение $n_{1}+\ldots+n_{m+1}=N$ в виде $n_{1}+\ldots+n_{m}=N-n_{m+1}$ и, заменив $N-n_{m+1}$ на $n$, запишем

$$
r_{m+1}(N)=\sum_{\substack{n_{m+1}=1,\left\{n_{m+1} \alpha_{m+1}\right\} \in I_{m+1}}}^{N-1} r_{m}\left(N-n_{m+1}\right)=\sum_{\substack{n=1,\left\{(N-n) \alpha_{m+1}\right\} \in I_{m+1}}}^{N-1} r_{m}(n) .
$$

При $k=m+1$ имеем $d=\operatorname{dim}_{\mathbb{Q}} \mathbb{A}^{m+1}$. Рассмотрим два случая: $d \geqslant 3$ и $d=2$. 
Пусть $d \geqslant 3$. Воспользуемся леммой 1 и перепишем (9) в виде

$$
r_{m+1}(N)=\sum_{\substack{n=1,\left\{n \alpha_{m+1}\right\} \in I_{m+1}^{\prime}(N)}}^{N-1} r_{m}(n)=\sum_{n=1}^{N-1} r_{m}(n) \chi_{I_{m+1}^{\prime}(N)}\left(\left\{n \alpha_{m+1}\right\}\right),
$$

где $I_{m+1}^{\prime}(N)=\iota_{\left\{N \alpha_{m+1}\right\}}\left(I_{m+1}\right)$.

В случае $d \geqslant 3$ найдутся такие числа $\alpha_{i}, \alpha_{j}$, что $1, \alpha_{i}, \alpha_{j}$ линейно независимы над $\mathbb{Q}$. Переобозначим числа $\alpha_{i}$ так, чтобы числа $1, \alpha_{1}, \alpha_{2}$ были линейно независимыми; тогда $\operatorname{dim}_{\mathbb{Q}} \mathbb{A}^{m} \geqslant 3$ и для $r_{m}(n)$ справедлива асимптотика (8), т.е.

$$
r_{m}(n) \sim \frac{1}{(m-1) !} \prod_{i=1}^{m}\left|I_{i}\right| \cdot n^{m-1} .
$$

Подставляя последнее выражение в формулу (9), получим асимптотику для $r_{m+1}$ :

$$
\sum_{n=1}^{N-1} \frac{1}{(m-1) !} \prod_{i=1}^{m}\left|I_{i}\right| \cdot n^{m-1} \cdot \chi_{I_{m+1}^{\prime}(N)}\left(\left\{n \alpha_{m+1}\right\}\right) \sum_{n=1}^{N-1} n^{m-1} \chi_{I_{m+1}^{\prime}(N)}\left(\left\{n \alpha_{m+1}\right\}\right) .
$$

Применим лемму 4 и запишем

$$
r_{m+1}(N) \sim \frac{1}{(m-1) !} \prod_{i=1}^{m}\left|I_{i}\right| \cdot \frac{\left|I_{m+1}^{\prime}\right|}{m}(N-1)^{m} \sim \frac{1}{m !} \prod_{i=1}^{m+1}\left|I_{i}\right| \cdot N^{m},
$$

так как $\left|I_{m+1}^{\prime}\right|=\left|I_{m+1}\right|$. Таким образом, теорема 1 доказана в случае $d \geqslant 3$.

Пусть $d=2$. Перепишем (9) в виде

$$
r_{m+1}(N)=\sum_{n=1}^{N-1} r_{m}(n) \chi_{I_{m+1}}\left(\left\{(N-n) \alpha_{m+1}\right\}\right) .
$$

В данном случае $\operatorname{dim}_{\mathbb{Q}} \mathbb{A}^{m}=2$, и для $r_{m}(n)$ справедлива асимптотика (8), т.е.

$$
r_{m}(n) \sim \frac{1}{(m-1) !} \rho_{m}\left(\left\{N \hat{\alpha}_{m}\right\}\right) n^{m-1} .
$$

Значит,

$$
\begin{aligned}
& r_{m+1}(N) \sim \frac{1}{(m-1) !} \sum_{n=1}^{N-1} \rho_{m}\left(\left\{n \hat{\alpha}_{m}\right\}\right) \cdot n^{m-1} \cdot \chi_{I_{m+1}}\left(\left\{(N-n) \alpha_{m+1}\right\}\right) \sim \\
& \sim \frac{1}{(m-1) !} \sum_{n=1}^{N-1} n^{m-1} \rho_{m}\left(\left\{n \hat{\alpha}_{m}\right\}\right) \chi_{I_{m+1}}\left(\left\{(N-n) \alpha_{m+1}\right\}\right) .
\end{aligned}
$$

В формуле суммирования по Абелю (5) положим

$$
\begin{gathered}
a_{n}=n^{m-1}, \quad b_{n}=\rho_{m}\left(\left\{n \hat{\alpha}_{m}\right\}\right) \chi_{I_{m+1}}\left(\left\{(N-n) \alpha_{m+1}\right\}\right), \\
B_{n}=\sum_{i=1}^{n} \rho_{m}\left(\left\{i \hat{\alpha}_{m}\right\}\right) \chi_{I_{m+1}}\left(\left\{(N-i) \alpha_{m+1}\right\}\right) ;
\end{gathered}
$$


тогда

$$
\begin{aligned}
r_{m+1}(N) \sim \frac{1}{(m-1) !}\left((N-1)^{m-1} B_{N-1}-\sum_{n=1}^{N-2}\left((n+1)^{m-1}-n^{m-1}\right) B_{n}\right)= \\
=\frac{1}{(m-1) !}\left((N-1)^{m-1} B_{N-1}-\sum_{n=1}^{N-2} B_{n}\left(\sum_{l=0}^{m-2} C_{m-1}^{l} n^{l}\right)\right) \sim \\
\sim \frac{1}{(m-1) !}\left((N-1)^{m-1} B_{N-1}-\sum_{n=1}^{N-2}(m-1) n^{m-2} B_{n}\right)
\end{aligned}
$$

Отдельно рассмотрим величину $B_{n}$, представляющую собой сумму произведений двух функций $\rho_{m}\left(\left\{i \hat{\alpha}_{m}\right\}\right)$ и $\chi_{I_{m+1}}\left(\left\{(N-i) \alpha_{m+1}\right\}\right)$.

Из условия $d=2$, согласно лемме 6 , следует, что $1, \hat{\alpha}_{m}, \alpha_{m+1}$ линейно зависимы над $\mathbb{Z}$. В этом случае замыкание множества точек $\left\{Q_{i}=\left(\left\{i \hat{\alpha}_{m}\right\},\left\{i \alpha_{m+1}\right\}\right)\right\}$, где $i$ - натуральное, называемое орбитой точек $Q_{i}$ и обозначаемое $\operatorname{Orb}\left(\hat{\alpha}_{m}, \alpha_{m+1}\right)$, будет являться объединением конечного числа отрезков, лежащих на параллельных прямых (см. [25]).

Далее, орбиту $\operatorname{Orb}\left(\hat{\alpha}_{m}, \alpha_{m+1}\right)$ можно отождествить с интервалом $[0 ; 1)$, склеив точки вида $(x, 1)$ с точками $(x, 0)$, а точки $(1, y)$ с точками $(0, y)$, и сжав полученный интервал до единичной длины. При таком отождествлении каждой точке $Q_{i}$ на орбите $\operatorname{Orb}\left(\hat{\alpha}_{m}, \alpha_{m+1}\right)$ будет соответствовать единственная точка $\left\{i \hat{\alpha}_{m+1}\right\}$ промежутка $[0 ; 1)$, где

$$
h a t \alpha_{m+1}=\frac{\left|\left(\hat{\alpha}_{m}, \alpha_{m+1}\right)\right|}{\left|\operatorname{Orb}\left(\hat{\alpha}_{m}, \alpha_{m+1}\right)\right|} \text {. }
$$

Построим такое соответствие $\kappa$, что $\kappa: \operatorname{Orb}\left(\hat{\alpha}_{m}, \alpha_{m+1}\right) \rightarrow[0 ; 1)$. Ясно, что отображение $\kappa$ взаимно однозначно и кусочно линейно. Определим на $[0 ; 1)$ функцию $\hat{\rho}_{m}(x)=\rho_{m}\left(x^{\prime}\right)$, где $x^{\prime}-$ абсцисса точки $\kappa^{-1}(x)$. Функция $\hat{\rho}_{m}(x)$ является кусочным многочленом степени $m-1$ как композиция кусочных многочленов степени $m-1$. Также определим множество $\hat{I}_{m+1} \subseteq[0 ; 1)$ как множество таких точек $y \in[0 ; 1)$, что ордината точки $\kappa^{-1}(y) \in I_{m+1}$. Множество $\hat{I}_{m+1}$ представляет собой объединение конечного числа интервалов, а его характеристическая функция - кусочно постоянна.

С учетом сказанного имеем

$$
B_{n}=\sum_{i=1}^{n} \hat{\rho}_{m}\left(\left\{i \hat{\alpha}_{m+1}\right\}\right) \chi_{\hat{I}_{m+1}}\left(\left\{(N-i) \hat{\alpha}_{m+1}\right\}\right)
$$

Согласно лемме 2 имеем

$$
B_{n} \sim n \int_{0}^{1} \hat{\rho}_{m}(t) \chi_{\hat{I}_{m+1}}\left(\left\{N \hat{\alpha}_{m}-t\right\}\right) d t
$$

Введем обозначение

$$
\rho_{m+1}(x)=\int_{0}^{1} \hat{\rho}_{m}(t) \chi_{\hat{I}_{m+1}}(x-t) d t, \quad m \geqslant 2 .
$$

Функция $\rho_{m+1}(x)$ является непрерывным кусочным многочленом степени $m$, где $m \geqslant 2$, так как удовлетворяет всем условиям леммы 12, и, в свою очередь согласно леммам 8, 9, 10, функция $\hat{\rho}_{2}(x)$ - непрерывная периодическая кусочно линейная функция, угловые коэффициенты которой принимают не менее двух различных значений. 
Перепишем $B_{n}$ в виде $n \rho_{m+1}\left(\left\{N \hat{\alpha}_{m+1}\right\}\right)$. Подставив это выражение в формулу (10), получим

$$
\begin{array}{r}
r_{m+1}(N) \sim \frac{1}{(m-1) !}\left((N-1)^{m} \rho_{m+1}\left(\left\{N \hat{\alpha}_{m+1}\right\}\right)-\sum_{n=1}^{N-2}(m-1) n^{m-1} \rho_{m+1}\left(\left\{N \hat{\alpha}_{m+1}\right\}\right)\right)= \\
=\frac{1}{(m-1) !} \rho_{m+1}\left(\left\{N \hat{\alpha}_{m+1}\right\}\right)\left((N-1)^{m}-(m-1) \sum_{n=1}^{N-2} n^{m-1}\right) .
\end{array}
$$

Воспользуемся асимптотикой (7) и получим

$$
\begin{gathered}
r_{m+1}(N) \sim \frac{1}{(m-1) !} \rho_{m+1}\left(\left\{N \hat{\alpha}_{m+1}\right\}\right)\left((N-1)^{m}-\frac{m-1}{m}(N-2)^{m}\right) \sim \\
\sim \frac{1}{(m-1) !} \rho_{m+1}\left(\left\{N \hat{\alpha}_{m+1}\right\}\right)\left(N^{m}-\frac{m-1}{m} N^{m}\right)= \\
=\frac{1}{(m-1) !} \rho_{m+1}\left(\left\{N \hat{\alpha}_{m+1}\right\}\right) \frac{1}{m} N^{m}=\frac{1}{m !} \rho_{m+1}\left(\left\{N \hat{\alpha}_{m+1}\right\}\right) N^{m} .
\end{gathered}
$$

Таким образом,

$$
r_{m+1}(N) \sim \begin{cases}\frac{1}{m !} \rho_{m+1}\left(\left\{N \hat{\alpha}_{m+1}\right\}\right) N^{m}, & \text { если } d=2, \\ \frac{1}{m !} \prod_{i=1}^{m+1}\left|I_{i}\right| \cdot N^{m}, & \text { если } d \geqslant 3,\end{cases}
$$

что совпадает с утверждением теоремы 1 при $k=m+1$. Теорема полностью доказана.

\section{СПИСОК ЛИТЕРАТУРЫ}

1. Гриценко С. А., Мотькина Н. Н. Задача Хуа-Локена с простыми числами специального вида// Докл. АН Респ. Таджикистан. - 2009. - 52, № 7. - С. 497-500.

2. Грищенко С. А., Мотькина Н. Н. О вычислении некоторых особых рядов// Чебышев. сб. - 2011. 12, № 4. - C. 85-92.

3. Гриценко С. А., Мотькина Н. Н. О некоторых аддитивных задачах теории чисел// Науч. вед. БелГУ. Сер. мат. физ. - 2010. -5 (76), № 18. - С. 83-87.

4. Гриценко C. А., Мотькина Н. Н. Об одном варианте тернарной проблемы Гольдбаха// Докл. АН Респ. Таджикистан. - 2009. - 52, № 6. - С. 413-417.

5. Гриценко С. А., Мотькина Н. Н. О теореме Чудакова в простых числах специального вида// Чебышев. сб. - 2011. - 12, № 4. - С. 75-84.

6. Гриценко С. А., Мотькина Н. Н. Проблема Варинга с натуральными числами специального вида// Чебышев. сб. - 2014. - 53, № 3. - С. 31-47.

7. Давлетлрова Е. П., Жукова А. А., Шутов А. В. Геометризация системы счисления Фибоначчи и ее приложения к теории чисел// Алгебра и анализ. - 2013. - 25, № 6. - С. 1-23.

8. Давлетлрова Е. П., Жукова А. А., Шутов А. В. Геометризация обобщенных систем счисления Фибоначчи и ее приложения к теории чисел// Чебышев. сб. - 2016. - 17, № 2. - С. 88-112.

9. Жукова А. А., Шутов А. В. Бинарная аддитивная задача с числами специального вида// Чебышев. сб. -2015 . - 16, № 3. - С. 247-275.

10. Журавлев В. Г. Гиперболы над двумерными квазирешетками Фибоначчи// Фундам. прикл. мат. 2010. - 16, № 6. - C. 45-62.

11. Журавлев В. Г. Четно-фибоначчевы числа: бинарная аддитивная задача, распределение по прогрессиям и спектр// Алгебра и анализ. - 2008. - 20, № 3. - С. 18-46.

12. Журавлев В. Г. Одномерные квазирешетки Фибоначчи и их приложения к диофантовым уравнениям и алгоритму Евклида// Алгебра и анализ. - 2007. - 19, № 3. - С. 151-182.

13. Журавлев В. Г. Суммы квадратов над о-кольцом Фибоначчи// Зап. науч. сем. ПОМИ. $-2006 .-337$. - C. $165-190$.

14. Журавлев В. Г. Разбиения Рози и множества ограниченного остатка// Зап. науч. сем. ПОМИ. -2005. - 322. - C. 83-106. 
15. Журавлев В. Г. Уравнение Пелля над о-кольцом Фибоначчи// Зап. науч. сем. ПОМИ. - 2007. -350. - C. 139-159.

16. Красильщиков В. В., Шутов А. В., Журавлев В. Г. Одномерные квазипериодические разбиения, допускающие вложение прогрессий// Изв. вузов. Мат. - 2009. - 7. - С. 3-9.

17. Красильщиков В. В., Шутов А. В. Распределение точек одномерных квазирешеток по переменному модулю// Изв. вузов. Мат. - 2012. - 3. - С. 17-23.

18. Шутов А. В. Арифметика и геометрия одномерных квазирешеток// Чебышев. сб. -2010 . -11 , № 1. - C. $255-262$.

19. Шутов А. В. Об одной аддитивной задаче с дробными долями// Науч. вед. БелГУ. Сер. мат. физ. 2013. - 5 (148), № 30. - С. 111-120.

20. Шутов А. В. Системы счисления и множества ограниченного остатка// Чебышев. сб. - 2006. -7 , № 3. - C. $110-128$.

21. Шутов А. В. Тригонометрические суммы над одномерными квазирешетками// Чебышев. сб. - 2012. - 13, № 2. - C. 136-148.

22. Akiyama S. Self affine tiling and Pisot numeration system// in: Number Theory and Its Applications (Gyory K., Kanemitsu S., eds.). — Kluwer, 1999. — P. 7-17.

23. Rauzy G. Nombres algb́riques et substitutions// Bull. Soc. Math. France. — 1982. — 110. — P. 147-178.

24. Shutov A. V., Maleev A. V., Zhuravlev V. G. Complex quasiperiodic self-similar tilings: their parameterization, boundaries, complexity, growth and similarities// Acta Crystallographica. - 2010. - A66. P. 427-437.

25. Weyl H. Üeber die Gleichverteilung von Zahlen mod. Eins.// Math. Ann. — 1916. — 77 (3). — P. 313-352.

Жукова Алла Адольфовна

Владимирский филиал Российской академии народного хозяйства и государственной службы при Президенте Российской Федерации

E-mail: georg967@mail.ru

Шутов Антон Владимирович

Владимирский государственный университет им. А. Г. и Н. Г. Столетовых

E-mail: a1981@mail.ru 